\title{
The Importance of Effective Collaboration Between Health Professionals for the Facilitation of Optimal Community Diabetes Care
}

\section{Jo Melville-Smith, Garth Kendall}

\begin{abstract}
Diabetes places a significant burden on the individuals concerned, their families, and society as a whole. The debilitating sequelae of diabetes can be limited or prevented altogether through strict glycaemic control. Despite the seemingly uncomplicated nature of the disorder, effective management can be elusive as the impact of having to deal with diabetes on a daily basis can be profound and appropriate professional support is not always readily available. As the roles of General Practitioners (GPs) and allied health professionals have evolved, a major issue now facing all is that of developing and maintaining effective collaborative relationships for the facilitation of optimal community diabetes care. Using a simple survey methodology, the present exploratory study investigated the referral patterns of GPs to Diabetic Educators (DEs) working for a Community Health Service in an Australian town and reasons for referral and nonreferral in order to identify factors that contribute to a sound and sustainable collaborative relationship. The results provide some evidence that GPs and DEs in this town do work collaboratively toward achieving client-centred goals and highlight the need to inform GPs who are new to communities, such as this one, of the available DE services. Most importantly, the study identified that there are many opportunities to strengthen collaboration so as to facilitate optimal community diabetes care. This information is valuable because there is limited empirical evidence either nationally or internationally
\end{abstract}


about the process of collaboration between health professionals in the management of chronic diseases, such as diabetes. 


\section{Introduction}

Diabetes is one of the fastest growing chronic health problems in Australia with a rapidly increasing incidence and prevalence, and high rates of mortality and morbidity (Booth, Cooper, \& Gill, 2008; Dunstan et al., 2002). Between 2000 and 2007 the rate of new cases of insulin-treated Type 2 Diabetes Mellitus (T2DM) increased from 69.3 to 96.2 per 100,000 (Australian Institute of Health and Welfare, 2009). In the same time period, the age-adjusted rate of new cases of Type 1 Diabetes Mellitus (T1DM) in children aged 0-14 years increased from 19 to 23 per 100,000, an average annual increase of 2.7 per cent per year (Faulks \& Flack, 2008). Diabetes not only places a burden on the individual concerned, but also their family and society as a whole. Common complications include: neuropathy, nephropathy, vision disorders, heart disease, stroke and peripheral vascular disease. Unsurprisingly, 30 per cent of people with diabetes have clinically relevant depression (Egede, 2005). Financial implications for families, such as medication and treatment costs and time off work due to incapacity or to accompany a child to medical appointments, can be significant (National Health and Medical Research Council, 2005). In Australia in 1999-2000 the annual cost to the nation was estimated to exceed \$1.2 billion, making it one of the most challenging public health issues (Cusack, Asyo, Frost, O’Brien, \& O’Kane, 2008).

While the long term effects of hyperglycaemia are damaging there is evidence to show that this can be ameliorated by controlling blood glucose levels (Bryant, 2006). Management goals are directed at reducing hyperglycaemia in order to prevent the debilitating microvascular and macrovascular complications that it can cause. The overall 
aim is to improve quality of life and prevent premature death (Harris, Mann, Marshall, Phillips, \& Webster, 2008). Despite the seemingly uncomplicated nature of the disorder, effective management can be elusive as the impact of having to deal with diabetes on a daily basis can be profound (Australasian Paediatric Endocrine Group, 2005) and appropriate professional support is not always readily available. Changes have recently been made in the way diabetes care is funded, not only in response to the growing number of people who develop diabetes, but also in view of the complexity of care these people require. A new Medicare initiative allows chronically ill people who are being managed by their General Practitioner (GP) under an Enhanced Primary Care Plan access to Medicare rebates for allied health services (Medicare Australia, 2005). GPs are now able to refer their clients to allied health practitioners, such as private Credentialled Diabetes Educators (CDEs) and dietitians, who then charge Medicare a fee for their service (Zwar, 2007). These changes have also impacted on DEs who offer diabetes management services within the public health sector. As the roles of GPs and allied health professionals have evolved, a major issue now facing all is that of developing and maintaining effective collaborative relationships for the facilitation of optimal community diabetes care. Collaboration in the health care setting involves attending to others' concerns while not sacrificing one’s own concerns. "The work of collaboration requires sharing control in an effort to obtain innovative solutions that are mutually acceptable” (Northhouse \& Northhouse, 1985), p. 306).

According to Harris (2008), in the team management of diabetes the client is the central member, with input from the GP, DE, dietitian, podiatrist, ophthalmologist or 
optometrist, oral health professional, exercise professional, and endocrinologist, diabetologist, or paediatrician. Incorporating a collaborative approach to diabetes care has been shown in several studies to produce favourable outcomes for the client (Desai et al., 2003; Taylor, Oberle, Crutcher, \& Norton, 2005; Wagner et al., 2001). A recent Australian study, for example, has shown that a collaborative, client focused approach towards diabetes care has impacted favourably on outcomes (Grimmer-Somers, Dolejs, Atkinson, \& Worley, 2008). The authors report on the evaluation of a chronic disease management program for clients with T2DM conducted in the Central Northern Adelaide Health Service, South Australia. In conjunction with four divisions of general practice the program was delivered by a team of providers including: GPs, practice nurses, CDEs, dieticians and podiatrists. The results of the program showed that there were benefits to the diabetes team and diabetes clients if integrated multidisciplinary care is provided.

The achievement of effective diabetes collaborative care invariably relies on all members of the diabetes team working together to achieve favourable outcomes. However, for the team approach to be successful there should be good communication between members based on trust and respect (Harris et al., 2008). This association has been explored by Pike (1991) who found that collaborative relationships between nurses and physicians can be grounded on mutual trust and respect with an appreciation that the two practice areas are interdependent, and the development of a synergistic alliance between the two can enhance client care. In a recent New Zealand study Pullon (2008) examined doctors' and nurses' perceptions of their own and each others' roles and the perceived relationships between individuals from both disciplinary groups. It was found that effective 
interprofessional relationships between individual doctors and nurses can, and often do, exist. Further, professional identity was related to the demonstration of professional competence and this in turn related to the development of mutual interprofessional respect and enduring interprofessional trust.

According to Eigenmann, Colagiuri, National Diabetes Services Scheme (Australia), \& Diabetes Australia (2007), DEs are the vanguard of providing diabetes client education. And yet they also state that the role of the team members, including the DE, is to augment the care delivered by the GP and to report appropriately care strategies addressed with the client. On the one hand it is inferred that DEs are team leaders, and on the other that DEs simply augment care given by the GP. The delineation of roles, especially the leadership role, is contenscious and it is likely to make the team approach to care difficult to negotiate. This can be further appreciated when considering the role of the $\mathrm{CDE}$ who is now able to bill Medicare for the delivery of diabetes services to eligible clients. CDEs have a degree of expertise regarding diabetes management which may at times bring them into conflict with GPs regarding optimal treatment methodologies. Other issues to consider within this framework include the medical model of diabetes care, which has traditionally been driven by the medical profession versus the client focused empowerment model, which is favoured by most DEs (Skinner et al., 2008). It is therefore not unreasonable to suggest that at times GPs may feel a degree of role erosion regarding the management of their diabetes clients. 
The present exploratory study investigated the referral patterns of GPs to DEs working for a Community Health Service in an Australian town and reasons for referral and nonreferral in order to identify factors that contribute to a sound and sustainable collaborative relationship between GPs and DEs and facilitate optimal community diabetes care.

The diabetes management service that is offered to clients by the Community Health Service is multifaceted and includes the provision of education and skills training with the aim of empowering and promoting self-sufficiency in the management of diabetes. An evidenced-based best practice approach is used in accordance with the latest national diabetes care guidelines (Eigenmann et al., 2007). A regular review service is offered to diabetes clients, either at the local hospital or in one of the GP's surgery, to assess their current glycaemic control. Support and reinforcement of self-care practices is provided and, after goals are identified, an agreed action plan is discussed. The action plan is an important component of self-care and it is done so that the client's can tackle their goals one-by-one in their order and at their own pace (Rodgers, 2008). The client's GP is notified of the client's visit, including the findings and any considerations which may enhance management. 


\section{Methods}

\section{$\underline{\text { Participants }}$}

All 19 GPs who were registered with the Australian Medical Board and currently delivering medical services in the town between April and June 2009 were invited to participate. The town, with a population of approximately 20,000, is a regional centre in Australia. The region experiences high socio-economic disadvantage relative to much of the population of the state, but has a similar demographic profile. The town and region is not named in this report to protect the confidentiality of GPs and the DE service.

\section{Procedure}

The listings of all GPs was obtained from the Hospital and Health Service. GPs were contacted by letter and asked to complete a survey and return it by pre-paid return addressed envelope within one month. If no response was obtained after, one month, a colleague of the researcher telephoned the surgery and asked the reception staff to, remind the GP, and/or, a colleague of the researcher visited the practice in person and requested the receptionist/practice nurse to remind the GP to complete the questionnaire. Several attempts were made by the researcher's colleagues to contact three remaining GPs to encourage them to respond, in an attempt to obtain a 100 per cent response rate. However, after a total period of three months it was decided to abandon further recruitment attempts. The study was approved by the Institutional Ethics Committee. 


\section{$\underline{\text { Measure }}$}

Both closed and open-ended questions were included in a three page questionnaire. To ensure that the instrument could be completed quickly by busy GPs most questions were either binary (Yes/No) or categorical and answered by selecting a tick box. However, three open-ended questions were included in order to generate depth and intensity where this essential information was required. The research questions were: How long has the participant been practising as a GP? How long has the participant been practising as a GP in town? Was the participant aware of the diabetes education services provided by the Community Health Service at the local Hospital? Would the participant like to know more about the diabetes education services provided by the Community Health Service at the local Hospital? Did the participant currently refer clients to the diabetes education services provided by the Community Health Service at the local Hospital? Was there any particular reason why clients are not referred for diabetes education services at the Community Health Service? Of those who refer their diabetes clients to the diabetes education services at the Community Health Service what were their reasons for dong this? Of those who refer their diabetes clients to the diabetes education services at the Community Health Service do they feel that the waiting time for seeing newly diagnosed diabetes clients by the $\mathrm{DE}$ is within reason? Of those who refer their diabetes clients to the diabetes education services at the Community Health Service did they believe clients get optimal management care from the DEs at the local Hospital? In the opinion of those who refer their diabetes clients to the diabetes education service Community Health Service could services be improved in any way? Would the participant like to discuss 
further the general management of diabetes with a DE from the Community Health Service?

\section{Data Analysis.}

A descriptive quantitative analysis was undertaken. The first phase of the analysis involved the derivation of dichotomous and categorical summary variables. Following re-coding these variables were tabulated using Statistical Package for Social Sciences (SPSS) software. Frequencies were then displayed in a summary table. The second phase of the analysis involved the derivation of categorical variables that summarised the data gathered in the open-ended questions. Significant statements, phrases and sentences were grouped to arrive at a number of categories for each variable. 


\section{Results}

\section{$\underline{\text { Response rate }}$}

A total of 16 out of a possible 19 GPs responded to the study, a response rate of 84 per cent.

\section{GP characteristics and referral patterns}

The majority of GPs practicing in the town (88\%), had over five years experience in general practice while the remainder had between two and five years experience. While 11 of the 16 respondents had practiced in the town for two or more years, the remaining five had practiced in the town less then two years and three of these GPs had practiced in the town for less than one year. All but two of the GPs reported that they knew about the DE service offered at the Community Health Service. The two GPs who were unaware had both practiced in the town for less than one year and both indicated that they would like to learn more about the DE service.

\section{$\underline{\text { Reasons for referral and non-referral }}$}

Of the 14 GPs who knew about the DE service 11 reported that they did refer their clients there. Reasons given for referral were: education give to clients is appropriate; no charge to client; clients requested the referral; client care plan provided; part of routine management; prompt scheduling time; and value support. Five of the 11 referring GP's believed that the current waiting time after triage was too long and eight believed that their clients got optimal diabetes care from the DE service. Of the three GPs who 
reported that they did not refer their clients, two indicated that they used a private CDE service and one did not respond.

\section{$\underline{\text { Suggestions for improvement of the DE service }}$}

Eight out of 11 GPs made suggestions for how, in their view, the service could be improved. Suggestions for improvement were: reduce waiting lists (two respondents); give clients more help to read labels (one respondent); provide a more supportive rehabilitation environment (one respondent); communicate with GPs using IT pathways, such as email (one respondent); provide clients with more educational material (one respondent); locate DE services in GP surgeries (one respondent); encourage clients to reduce time between their visits to the GP (one respondent); and increased follow-up of clients (one respondent).

Desire to discuss further the management of diabetes

Only one of the GPs who used the DE service indicated that they would like to discuss further the general management of diabetes with a DE from the Community Health Service. 


\section{Discussion}

The results provide evidence that the majority of GPs in this town do refer their clients to the DE service and acknowledge the role and relevance of the DE as a member of the diabetes management team. The findings have also demonstrated that predominately these GPs recognise that the care given by the DEs is suitable and that they rely on the service as an integral part of ensuring optimal diabetes care for their clients. Further positive inferences can be extrapolated from the fact that three survey responses indicated that the client requested the referral and, 'Other' comments made by GPs such as “ For care plan”, "Part of routine diabetes management', "I and clients value the support and input provided to the diabetic shared care management", "Prompt scheduling time” and

“In general the diabetes services are very good”. The results of the study will be discussed in more detail in the following paragraphs, with reference to the research literature, where appropriate.

The results highlight the need to inform GPs who are new to communities, such as this one, of the available DE services. While there is no research literature that specifically addresses the issue of establishing collaborative relationships with new GPs with regard to the management of diabetes, the way new GPs see their role has recently been explored. In a study of resident medical officers from three countries in their last year of training Beaulieu et al. (2009) found that respondents shared common conceptions of the family physician's role and that continuity of care and client advocacy were seen as the foundations of the discipline. Jones and Green (2006) followed the early careers of 20 GPs and found that the cohort was characterised by a change in professionalism away 
from values attributed to traditional general practice to a less paternalistic forms of relationships with clients which may then be realised in healthcare delivery. This trend for a change in GP attitude away from traditional models of care is well cemented in the concept of GPs being part of diabetes collaboration rather than having a traditional didactic approach.

As the project included open-ended questions, rich data were generated and post hoc analysis resulted in several issues being illuminated. GPs suggestions for improvement which were summarised from the analysis are interpreted as useful feedback as part of enhanced communication, rather than negative criticism. Professional collaboration involves open and honest communication with information being delivered in a positive and supportive manner (Hiss, Armbruster, Gillard, \& McClure, 2007).

A number of GPs indicated that waiting times following referral to the DE service were excessive and this was the most common response to the question asking how services could be improved. Primary health care services in regional areas generally have long waiting times which suggests they are under-staffed and under-funded. In a recent study involving T2DM clients in another Australian regional centre, it was found that participants experienced difficulties in gaining access to quality services and they experienced long waiting times (Wellard, Rennie, \& King, 2008). Similarly, a reduction in waiting times was one of the main areas of concern by health care professionals, clients and carers in a study of diabetes services in rural and remote areas of Scotland (Cramp, 2006). There is little doubt that increased funding and more staff will be 
required to address the dramatic worldwide increase in the prevalence of both T1DM and T2DM (Shaw \& Chisolm, 2003). While DEs are able to consolidate client selfmanagement skills needed to adequately address the disease (Harris et al., 2008), effective management takes time (Paterson, 2001). GPs and DEs who are at the "coal face” of diabetes care are in a unique position to be able to collaborate to advocate for increased funding for diabetes services.

Another issue raised, was the absence of communication using email and other IT-based approaches rather than the current practice of sending a letter. It is assumed that email would make communication easier for both parties and facilitate more timely correspondence. While there is no research literature that addresses this specific issue, Berendsen et al. (2009) did assess the procedural aspects of communication between GPs and specialists using telephone and letter, and most recently, email. Findings revealed that GPs and specialists disagree on several aspects of their communication which can impede improvements in client care. The authors suggest that GPs and specialists should discuss amongst themselves how best to compose a format for the referral letter, and the specialist's report, and how to go about exchanging mutual feedback. This study highlighted the need to develop mutually agreed communication systems and the fact that the capacity to do this may be improved as technology advances.

In the current context it is not possible for GPs and DEs to communicate confidential client information by email because there is a risk it may be intercepted by a third party. However, as technology continues to advance this capability may well become available. 
Another possibility considered by the National Health and Hospitals Network (2010) in Australia as a way of making information exchange more efficient and improving collaboration between health care providers is a hand-held electronic device kept by the client into which the service providers could input information .

A further suggestion was made by GPs in relation to providing enhanced support and encouragement to diabetes clients. These comments indicate that GPs acknowledge that diabetes clients require support and encouragement to cope with their disease and that DEs are often in a better position to be able support clients to manage appropriately (Harris et al., 2008; Hill \& Clark, 2008). Following on from this is the GP's recognition of the importance of providing follow-up and the timing of visits. At the Community Health Service in question, diabetes clients do have the opportunity to be periodically reviewed by the DEs and a recall system is used to initiate client reviews. Newly diagnosed clients are offered a follow up review at three to six months after diagnosis, depending on degree of diabetes control, and again at 12 months time. Those clients with elevated glycated haemoglobin levels are also followed up periodically for continuing assessment and assistance with diabetes management as required. The recognition by GPs of the need for DE follow-up is another example of a common understanding that will facilitate collaboration and optimal multidisciplinary care.

Finally, one GP suggested relocating DE services to GP practices. This idea is in keeping with proposed changes to health care policy and practice in response to theories of chronic disease management (Wagner et al., 2001) recently outlined in World Health 
Organisation (WHO) guidelines (Chew, 2009). WHO have recommended a move away from a reactive system that focuses on acute care, towards a more proactive approach that supports the management of chronic disease (Chew, 2009). Changes in the management of chronic diseases, such as diabetes, have been considered for some time and there is some evidence that a collaborative approach to diabetes care in the primary care setting is beneficial for both clients and heath care professionals (Reed, Revel, Carter, Saadi, \& Dunn, 2001; Sturmberg \& Overend, 1999). The Australian Federal Government has responded to this call to implement changes in chronic care service delivery by instigating a 'National Primary Health Care Strategy' initiative which aims to refocus health care delivery within Australia (Department of Health and Aging, 2009). Key initiatives include: better rewards for primary health professionals for preventive interventions; the promotion of evidence-based management of chronic disease; supporting clients with chronic disease to manage their condition; supporting the role GPs play in the health care team; addressing the growing need for access to other health professionals, including practice nurses and allied health professionals, such as physiotherapists and dieticians; and the encouragement of a greater focus on multidisciplinary team-based care (Department of Health and Aging, 2009).

As a result of this initiative, the South Australia Department of Health, for example, has promoted the establishment of several "GP Plus Health Care Centres" throughout the state. These centres have been developed to help "South Australians take control of their health care, stay healthy and out-of-hospital” (South Australian Department of Health, 2009). The scheme has resulted in CDEs and other allied health professionals conducting 
services within GP surgeries rather than in hospital or community health settings. The service providers are able to practice privately and as previously discussed, bill Medicare Australia for services rendered, or they can be employed by a public funded health service.

A limitation of this project is that not all GPs participated. Several attempts were made to recruit the remaining three GPs, however, they chose not to engage in the survey. As the population of the town is small, any reduction in project number size may exert some influence on the results. For example, it is not known if the three non-respondents valued the DE service or not. Another limitation was the time constraint associated with the attempt to engage with GPs, as they are notoriously busy (Kaner, Haighton, \& McAvoy, 1998). To remedy this, the survey tool was designed to be as time efficient as possible with three open-ended questions to promote the addition of more meaningful data for depth and substance. Whilst questionnaires which are constructed to promote participant response by way of ease of completion, can be useful, there are a number of disadvantages. Firstly, despite the open-ended questions there is some loss of depth of information. Secondly, there was no opportunity for the research to clarify the comments made by some GPs. This is a common problem when using a self-administered questionnaire approach (Polger \& Thomas, 1995). While an attempt was made to further engage the GPs by asking if they would like to be contacted, it might have been better to have made an appointment with each one upon completion of the questionnaire. Thirdly, respondents may feel frustrated when answering closed response formats as the answers offered do not meet the responses they wish to convey (Polger \& Thomas, 1995). 
In conclusion, this exploratory study has identified that the majority of GPs in this community do refer their clients to the DE service and acknowledge the role and relevance of the DE as a member of the diabetes management team. Perhaps more importantly, a number of GPs identified opportunities to strengthen collaboration so as to facilitate optimal community diabetes care. This information is valuable because there is limited empirical evidence either nationally or internationally about the process of collaboration between health professionals in the management of chronic diseases, such as diabetes. We assert that a great deal more research is required to establish a firm evidence-base for interprofessional collaboration in health care. Any improvements that can be made are likely to be of great benefit to people with chronic diseases, to their families, and society as a whole. 


\section{References}

Australasian Paediatric Endocrine Group. (2005). Clinical practice guidelines: Type 1 diabetes in children and adolescents. . Canberra: National Health and Medical Research Council.

Australian Institute of Health and Welfare. (2009). Insulin-treated diabetes in Australia 2000-2007. Canberra: Australian Institute of Health and Welfare.

Beaulieu, M., Dory, V., Pestiaux, D., Pouchain, D., Rioux, M., Rocher, G., et al. (2009). What does it mean to be a family physician? Exploratory study with family medicine residents fro 3 countries. Canadian Family Physician,, 55, 14.

Berendsen, A., Kuiken, A., Benneker, W., Meyboom-de Jong, B., Voorn, T., \& Schuling, J. (2009). How do general practitioners and specialists value their mutual communication? A survey. BMC Health Services Research,, 9, 143.

Booth, K., Cooper, H., \& Gill, G. (2008). A trial of empowerment-based education in type 2 diabetes-Global rather than glycaemic benefits. Diabetes Research and Clinical Practice, 82, 165-171.

Bryant, W. (2006). What does 'diabetes guidelines’ mean in practice? . Diabetes Management, 17, 24.

Chew, D. (2009). A comparative study of the implementation of the Chronic Disease Management (CDM) System for diabetes in primary care. Australian Diabetes Educator, 12(4), 26.

Cramp, G. (2006). Development of an integrated and sustainable rural service for people with diabetes in the Scottish Highlands. Rural \& Remote Health, 6(1), 422.

Cusack, M., Asyo, N., Frost, C., O’Brien, K., \& O’Kane, G. (2008). Does intervention by the dietitian and diabetes educator of a More Allied Health Services program improve glycosylated haemoglobin levels those with type 2 diabetes? Implications for rural dietetic practice. Nutrition \& Dietetics, 65(292-329).

Department of Health and Aging. (2009). Building a 21st Century Primary Health Care System: A Draft of Australia's First National Primary Health Care Strategy. Canberra: Commonwealth of Australia.

Desai, J., Solberg, L., Clark, C., Reger, L., Pearson, T., Bishop, D., et al. (2003). Improving diabetes care and outcomes: the secondary benefits of a public healthmanaged care research collaboration. Journal of Public Health Management \& Practice, Suppl, S36-43.

Dunstan, D., Zimmet, P., Welborn, T., de Courten, A., Cameron, A., Sicree, A., et al. (2002). The rising prevalence diabetes and impaired glucose tolerance. The Australian diabetes, obesity and lifestyle study. Diabetes Care, 25(5), 829-834.

Egede, L. E. (2005). Effect of depression on self-management behaviors and health outcomes in adults with type 2 diabetes. Current Diabetes Reviews, 1(3), 235243.

Eigenmann, C., Colagiuri, R., National Diabetes Services Scheme (Australia), \& Diabetes Australia. (2007). Outcomes and indicators for diabetes education : a national consensus position. Canberra: National Diabetes Services Scheme, Diabetes Australia.

Faulks, K., \& Flack, J. (2008). Type 1 diabetes is increasing in Australia. Diabetes Management, 25, 24. 
Grimmer-Somers, K., Dolejs, W., Atkinson, J., \& Worley, A. (2008). Integrated GP and allied health care for patients with type 2 diabetes. Australian Family Physician, 37(9), 774-779.

Harris, P., Mann, L., Marshall, P., Phillips, P., \& Webster, C. (Eds.). (2008). Diabetes management in general practice 2008/2009 (14th ed.). Norah Head, NSW: Pinnacle Print.

Hill, P., \& Clark, R. (2008). The Australian DE's skills and readiness for the tsunami of diabetes in the 21st century. Australian Journal of Advanced Nursing, 26(2), 5565.

Hiss, R., Armbruster, B., Gillard, M., \& McClure, L. (2007). Nurse care manager collaboration with community-based physicians providing diabetes care: a randomized controlled trial. Diabetes Educator, 33(3), 493-502.

Jones, L., \& Green, J. (2006). Shifting discourses of professionalism:a case study of general practitioners in the United Kingdom. Sociology of Health \& Illness, 28(7), 927-950.

Kaner, E. F., Haighton, C. A., \& McAvoy, B. R. (1998). 'So much post, so busy with practice--so, no time!': a telephone survey of general practitioners' reasons for not participating in postal questionnaire surveys. British Journal of General Practice, 48(428), 1067-1069.

Medicare Australia. (2005). Allied Health Initiative. Retrieved August 28th, 2008, from http://www.medicareaustralia.gov.au/provider/incentives/allied-health.jsp

National Health and Hospitals Network. (2010). A National Health and Hospitals Network for Australia's Future - Delivering better health and better hospitals (No. 6620). Canberra: Commonwealth of Australia.

National Health and Medical Research Council. (2005). Clinical practice guidelines: Type 1 diabetes in children and adolescents. Canberra: Commonwealth of Australia.

Northhouse, P. G., \& Northhouse, L. L. (1985). Health communication: a handbook for health professionals. Englewood Cliffs, New Jersey: Prentice-Hall Inc.

Paterson, B. (2001). Myth of empowerment in chronic illness. Journal of Advanced Nursing, 34(5), 574-581.

Pike, A. (1991). Moral outrage and moral discourse in nurse-physician collaboration. Journal of Professional Nursing, 7(6), 351-362.

Polger, S., \& Thomas, S. (1995). Introduction to Research in the Health Science (3rd ed.). London: Churchill Livingstone.

Pullon, S. (2008). Competence, respect and trust: key features of successful interprofessional nurse-doctor relationships. Journal of Interprofessional Care, 22(2), 133-147.

Reed, R., Revel, A., Carter, A., Saadi, H., \& Dunn, E. (2001). A clinical trial of chronic care diabetic clinics in general practice in the United Arab Emirates: a preliminary analysis. Archives of Physiology \& Biochemistry, 109(3), 272-280.

Shaw, J. E., \& Chisolm, D. J. (2003). Epidemiology and prevention of type 2 diabetes and the metabolic syndrome. Medical Journal of Australia, 179, 379-383.

Skinner, T. C., Carey, M. E., Craddock, S., Dallosso, H. M., Daly, H., Davies, M. J., et al. (2008). "Educator Talk' and patient change: some insights from the DESMOND 
(Diabetes Education and Self Management for Ongoing and Newly Diagnosed) randomised controlled trial. Diabetic Medicine, 25(9), 1117-1120.

South Australian Department of Health. (2009). Better health for people through GP Plus Health Care Centres. Retrieved 2009, 5th October, from http://www.health.sa.gov.au/Default.aspx?tabid=265

Sturmberg, J., \& Overend, D. (1999). General practice based diabetes clinics. An integration model. Australian Family Physician, 28(3), 240-245.

Taylor, K., Oberle, K., Crutcher, R., \& Norton, P. (2005). Promoting health in type 2 diabetes: nurse-physician collaboration in primary care. Biological Research for Nursing, 6(3), 207-215.

Wagner, E., Glasgow, R., Davis, C., Bonomi, A., Provost, L., McCulloch, D., et al. (2001). Quality improvement in chronic illness care: a collaborative approach. Joint Commission Journal on Quality Improvement, 27(2), 63-80.

Wellard, S., Rennie, S., \& King, R. (2008). Perceptions of people with type 2 diabetes about self-management and the efficacy of community based services. Journal for the Australian Nursing Profession, 29(2), 218-226.

Zwar, N. (2007). General Practice Incentives and Diabetes Care. Diabetes Management Journal, 19, 24. 